\title{
Strength analysis of metallic bonded joints containing defects
}

\author{
Wei $\mathrm{Xu}^{*}$, Yueguang Wei \\ State Key Laboratory of Nonlinear Mechanics, Institute of Mechanics, Chinese Academy of Sciences, Beijing 100190, PR China
}

\section{A R T I C L E I N F O}

\section{Article history:}

Received 6 June 2011

Received in revised form 28 August 2011

Accepted 8 September 2011

Available online 22 October 2011

\section{Keywords:}

Finite element analysis

Cohesive zone model

Adhesively bonded joint

Defect

\begin{abstract}
A B S T R A C T
Finite element simulation has been utilized to study the overall strength of metallic single lap joints with defects in their adhesive layers. Three types of defects are taken into account respectively, which are local debonding, weak bonding and void. For the first two types of defects, a developed numerical method using the cohesive zone model modified by user-defined subroutines is carried out as to consider the influences of the defect size and location. Furthermore, a modified-Gurson model is employed to simulate the adhesive layer with voids, considering the influence of the void size. The results show that the overall strength of the joints diminishes as the defect size is increased. Especially, the adhesive fracture properties and the size of the weak bonding region have combined influences on the strength of the joints.
\end{abstract}

Crown Copyright (c) 2011 Published by Elsevier B.V. All rights reserved.

\section{Introduction}

Adhesively bonded joints are economical, practical and easy to make [1], thus they have been widely used in a variety of industries for connecting dissimilar materials, such as civil engineering [2-4], automotive [5-7] and aircraft industries [8]. However, one of the limitations for adhesively bonded joins is the difficulty in predicting joint strength after manufacture and during in-service operation due to the presence of defects in the adhesive [9]. The defects generated by inadequate preparation of the joints or environmental degradation (e.g., moisture effects) of the adhesive [9] would reduce the bonding quality and influence the joint strength accordingly [10].

Previous researchers have already developed some experimental methods to detect and evaluate the defects in the adhesives of the joints. Heslehurst [11] used the technology of holographic interferometry to study the structural response of bondline defects. The defect regions could be indicated by the interferogram fringe patterns. Yang et al. [12] implemented a nondestructive detection that was vibration damping and frequency measurement to evaluate the defects in composite joints. Llopart et al. [13] also carried out a nondestructive test on the bonded specimens by means of ultrasonic C-scan and X-ray imaging, for a purpose of assuring that the adhesive had spread out to the full bonding surface. Similarly, Tserpes et al. [14] also employed ultrasonic C-scan to check the quality of the bondline in joints, the existence of the defects (e.g., voids) could be inspected easily by the C-scan images. Since the voids are harmful to the reliability of the adhesively

\footnotetext{
* Corresponding author. Tel.: +86 10 82543954; fax: +86 1062561284 .

E-mail address: xuwei@Inm.imech.ac.cn (W. Xu).
}

bonded joints, it is of significance to assess the residual strength of joints when defects are detected.

Experimental studies for the residual strength of the joints with voids have been carried out by many groups $[10,11,15,16]$, meanwhile, numerous models for the adhesively bonded joints with defects have been developed, which may be grouped into two main categorizes: one is theoretical models based on some assumptions. Olia and Rossettos [17] proposed an analytical solution for a twodimensional plane strain problem of adhesively bonded joints with gap defects in adhesive. They pointed out that the stresses in the adhesive could be affected obviously when the defect was sufficiently close to the end of the overlap. Chadegani and Batra [18] used the first-order shear deformation theory (FSDT) to analyze stresses and displacements in an adhesively bonded joint with an interfacial crack and a void. They regarded both the adhesive and the adherends as linear elastic and homogeneous materials in the theoretical model. They concluded that the results agreed with that of the numerical model they carried out. The other category of models is numerical models employing some calculation method such as finite element analysis (FEA). In contrast with theoretical models, numerical models are easier for implementation with less assumptions, thus they have been employed widely in studying adhesively bonded joints with defects. Chow and Woo [19] studied the effects of internal flaw size and its distribution along the adhesive layer on the fracture behavior of an adhesively bonded joint by utilizing a FEA model. They concluded that defect in the adhesive layer had definite effect on the shear distribution and on the fracture strength of the joint. de Moura et al. [9] simulated the composite bonded joints with strip defects. The interface elements considering mixed-mode damage were introduced to calculate the stresses and damage initiation and growth in the adhesive. The results showed the specified strength 
of the joints was not affected by the size of the defect. You et al. [20] studied the influence of the strip defect on stress distribution in both the mid-bondline and the adherend near the interface along the lap zone of adhesively bonded joint. Ribeiro et al. [21] also developed two-dimensional and three-dimensional FEA models to model the stress distributions in single-lap adhesively joints with different types of defects. The simulation result showed the stresses near the defects and the glue extremities would be larger, and the defects placed near to the extremities significantly reduced the joint strength.

Although the mechanical behaviors of adhesively bonded joints with defects have been investigated by plenty of researchers, systematical results taking various types of defects into account are lacking according to our literature search. Moreover, most models concerned the influence of defects on the stress distributions in adhesives instead of the overall strength of joints. Besides, the defects in most numerical models were introduced by modeling configurations of discontinuous adhesives, which need to be meshed densely in the regions near the defects so as to improve the calculation accuracy. Sometimes it would be complicated and inefficient way to achieve the modeling defects. To address these deficiencies, in the present research, an alternative numerical model utilizing finite elements method is established to describe the mechanical behavior of the metallic lap joints with defects, focusing on the overall strength of the joints. Three types of defects are taken into account, which are local debonding, weak bonding and voids. For the first two types of defects, a developed numerical method using the cohesive zone model modified by user-defined subroutines is carried out as to consider the influences of the defect size and position. Furthermore, the Gurson-Tvergaad-Needleman (GTN) model is employed to simulate the adhesive layer with voids, considering the influence of the void size. Obtained results provide fundamental principles for factual strength assessment of adhesively bonded joints with defects in their adhesives.

\section{Simulation approach}

In this section, a numerical model of the single lap joint is built with the commercially available FEM code ABAQUS. In practice, the width of the sheets used for the joints is far larger than their thickness, thus the model can be simplified as a plane strain model with the consideration of saving computational resources without losing accuracy.

Fig. 1a depicts the computational model of the single lap joint, which consists of two same metal sheets with the thickness of $h$ and the length of $a(=40 h)$. The sheets are connected by an adhesive layer with the length of $l$, which can be also called overlap length. The sheets are meshed using four-node quadrilateral plane strain elements, of which the total number is set at 1920 upon checking the convergence of the numerical results. Under uniaxial stretching, the joint is taken to deform under plane strain. Along the left side of the joint, the horizontal displacement is set to be zero, whereas a uniform displacement of $u$ is applied to the right side of the joint.

The metal sheets are modeled as elastic-plastic solids, with their true stress versus strain curves fitted using power-law hardening laws [22], as:

$\sigma= \begin{cases}E \varepsilon & \varepsilon \leqslant \sigma^{Y} / E \\ \sigma^{Y}\left(\frac{\varepsilon}{\sigma^{Y} / E}\right)^{N} & \varepsilon>\sigma^{Y} / E\end{cases}$

where $E$ is the Young's modulus, $N$ is the strain hardening exponent, and $\sigma^{Y}$ is the yield strength. For the present model, the three material properties are $70 \mathrm{GPa}, 0.02$ and $275 \mathrm{MPa}$, respectively, taken from Ref. [23].
As depicted in Fig. 1a, the region highlighted by rectangular dashed box is termed overlap, which contains the adhesive with defects. Fig. $1 \mathrm{~b}$ displays the overlap regions with three types of defects respectively, which are local debonding, weak bonding and voids. In the following text, various interface elements would be implemented to simulate the adhesive with those defects. For a purpose of obtaining better computational accuracy, the overlap region is meshed densely while sparse mesh is adopted in other regions as shown in Fig. 1c.

\section{Local debonding}

\subsection{Interface elements}

Cohesive zone models (CZMs) based on traction laws are well suitable to describe the decohesion in composite structures. The CZMs require traction-separation $(\mathrm{T}-\mathrm{S})$ relations for characterizing the constitutive laws of them. So far, considerable researches have focused on the constitutive laws of CZMs and their applications [24]. It has been established that whilst the peak value and area of the T-S curve are vital for capturing the interface separation behavior, its precise shape is of much lesser significance [25]. Consequently, for simplicity, the bilinear T-S law [24,26,27] shown in Fig. 2 is selected for the present study. Built upon the bilinear cohesive zone model (CZM), the adhesive, also treated as interface between the two metal sheets, is modeled with the cohesive zone elements.

Fig. 2 presents the traction-separation (T-S) relation of the CZM, with Fig. 2a and b representing the relations in tensile and shear direction respectively. To distinguish the tensile T-S law from the shear one, let the superscript " $n$ " represent the normal direction and "s" denote the shear direction. In Fig. 2, $u_{m}$ and $u_{c}$ are the maximum and critical separation, respectively, and $T$ is the traction stress.

Since the maximum value of $T^{n}$ is $\sigma_{m}$ while that of $\left|T^{s}\right|$ is $\tau_{m}$, the interfacial fracture energies in the two directions can be expressed as:

$$
\begin{aligned}
\Gamma^{n} & =\int_{0}^{u_{m}^{n}} T^{n} d u^{n}=\frac{1}{2} \sigma_{m} u_{m}^{n} \\
\Gamma^{s} & =\int_{0}^{u_{m}^{s}} T^{s} d u^{s}=\frac{1}{2} \tau_{m} u_{m}^{s}
\end{aligned}
$$

As the loading is increased beyond a critical value, the interface begins to soften and degrade, namely, the interface is now in the damaged (or softening) state. Typically, damage is initiated when a certain criterion is satisfied. In the present study, inspired by the bilinear law of Fig. 2, the quadratic nominal stress criterion is adopted to characterize the interfacial damage, described as:

$$
\left(\frac{\left\langle T^{n}\right\rangle}{\sigma_{m}}\right)^{2}+\left(\frac{T^{s}}{\tau_{m}}\right)^{2}=1
$$

where \langle\rangle represents the Macaulay bracket defined by $\langle x\rangle=1$ / $2(x+|x|)$, with the usual interpretation that a pure compressive deformation or stress state does not initiate damage.

It is assumed that interfacial damage occurs when Eq. (3) is satisfied and a single damage variable $D$ based on the total displacement jump $\Delta$ is introduced (i.e., $\Delta=\sqrt{\left\langle u^{n}\right\rangle^{2}+\left(u^{s}\right)^{2}}$ ) [28,29], as:

$D=\frac{\Delta_{f}\left(\Delta_{\max }-\Delta_{c}\right)}{\Delta_{\max }\left(\Delta_{f}-\Delta_{c}\right)}$

where $\Delta_{c}$ and $\Delta_{f}$ denote the total displacement at damage initiation and complete failure. $\Delta_{f}$ is determined by $\Delta_{f}=2 \Gamma / T_{c}^{\text {eff }}$ with $T_{c}^{\text {eff }}$ as the effective traction at damage initiation (i.e., 


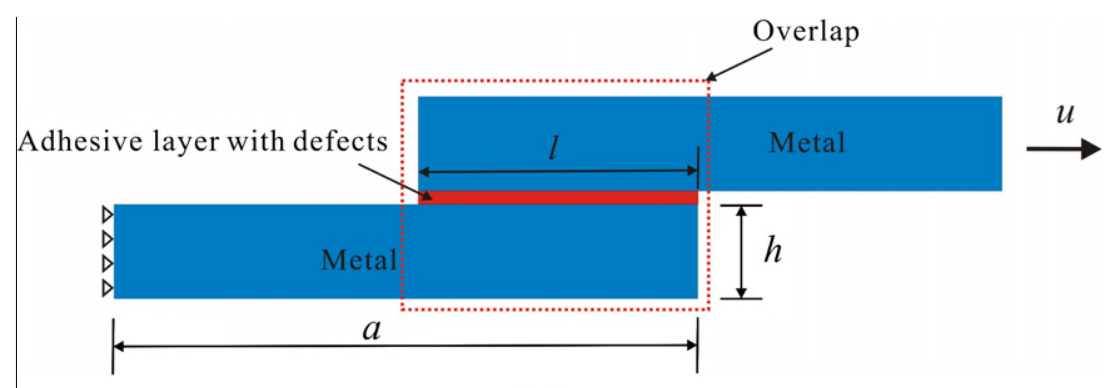

(a)

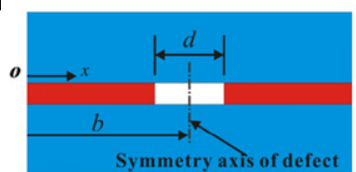

Local debonding

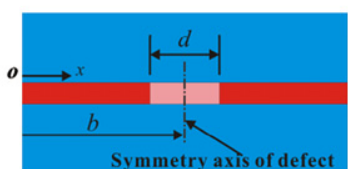

Weak bonding

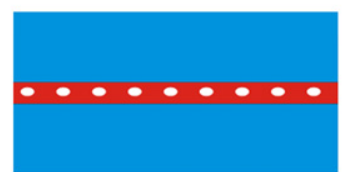

Voids

(b)

(c)

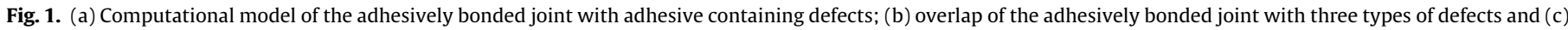
finite element mesh of the computational model.
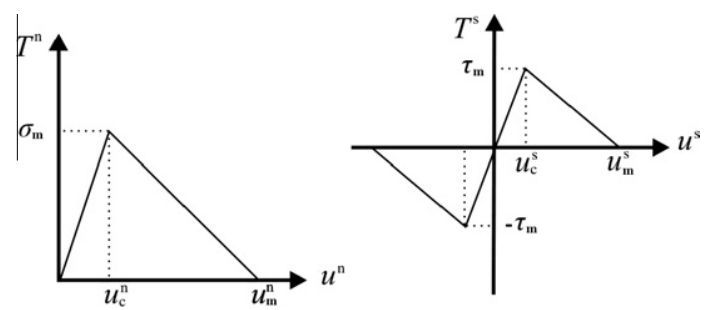

Fig. 2. Typical bilinear traction-separation law of cohesive zone model: (a) traction-separation relation in tension; (b) traction-separation relation in shear.

$\left.T_{c}^{\mathrm{eff}}=\sqrt{\left(T_{c}^{n}\right)^{2}+\left(T_{c}^{s}\right)^{2}}\right)$. In Eq. (4), $\Delta_{\max }$ denotes the maximum total displacement ever experienced during the loading history.

Noting that $\Gamma$ is the total interfacial fracture energy, it can be also seen as the fracture energy of the adhesive. Generally, the interface fracture energy $\Gamma$ depends on the mode-mixity. In other words, the interface fracture energy varies as a function of the mode-mixity. Thus the interface fracture energy could be termed the mixed-mode fracture energy. In the present investigation, the linear fracture criterion is employed to describe the mixed-mode, which could be expressed as,

$\frac{G^{n}}{\Gamma^{n}}+\frac{G^{s}}{\Gamma^{s}}=1$

where $G^{n}$ and $G^{s}$ denote work done by the traction and its conjugate relative displacement in the normal and shear directions, respectively. $\Gamma^{n}$ and $\Gamma^{s}$ refer to the critical fracture energies required to cause failure in the normal and shear directions, respectively, with the definition in Eq. (2). Consequently, the total interface fracture energy could be determined by $\Gamma=G^{n}+G^{s}$ when Eq. (5) is satisfied. It should be mentioned a major assumption is proposed in the present simulation, namely, $\Gamma^{n}=\Gamma^{s}$. By this assumption, the interface fracture energy $\Gamma$ is a constant in the present simulation.

Moreover, it is worth mentioning that the peak traction stresses $\sigma_{m}^{n}$ and $\tau_{m}^{s}$ are also called the separation strengths. Strictly speak- ing, the separation strength is different from the yield strength. In practice, however, the separation strength is sometimes treated equal to the yield strength of the adhesive when evaluating the strength of the adhesive bonding system [23]. Accordingly, the same approach is followed in the present study and the two peak traction stresses are assumed equal (i.e., $\sigma_{m}^{n}=\tau_{m}^{s}=\sigma_{a d}=8.27 \mathrm{MPa}$ ) [23].

The adhesive layer containing defect is modeled with a single layer of four-node modified cohesive elements, which share nodes with the neighboring elements in the upper and lower sheets. The defect is first considered as the form of local debonding, which means the interface elements lose the capability of connecting the sheets in some positions of the adhesive layer. In the present investigation, the local debonding is introduced by the user subroutine USDFLD of ABAQUS, which stands for user subroutine to redefine field variables. The subroutine is employed to define the distribution of the total fracture energy $\Gamma$ along the adhesive layer, which can be expressed as the function of the horizontal coordinate $x$ initiated at the left extremity of the adhesive layer (see, Fig. 1b),

$\Gamma=\Gamma(x)= \begin{cases}0 & x \in\left(b-\frac{1}{2} d, b+\frac{1}{2} d\right) \\ \Gamma_{0} & x \notin\left(b-\frac{1}{2} d, b+\frac{1}{2} d\right)\end{cases}$

where $\Gamma_{0}$, with a typical value of $1 \mathrm{~N} / \mathrm{mm}$, is the fracture energy of interface elements in the bonding area.

\subsection{Influence of defect size}

The influence of defect size on the load-bearing capacity has been investigated by the present numerical model. In this section, the center of the strip-defect coincides with the center of the adhesive. In other words, the strip-defect locates at the mid-region of the adhesive layer.

Fig. 3 displays the normalized load-displacement curves considering various sizes of debonding in the adhesive. In order to present the discrepancy between the cases with and without de- 


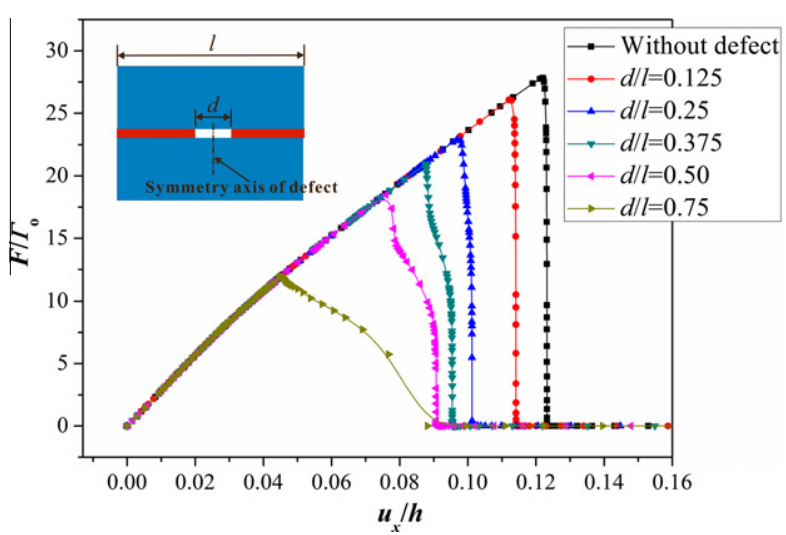

Fig. 3. Normalized load plotted as the function of displacement for the cases considering the selected normalized defect sizes $d / l$.

fects, the curve corresponding to perfect bonding is hence shown in the figure. All the curves present linear rising up at the beginning, which can be seen as the first stage of the deformation. However, the curves drop down after the loads arrive to peak values, which can be used to assess the load-bearing capacity of the adhesively bonded joints [30]. It is interesting in Fig. 3 that not only the peak values exhibit discrepancy but also the curves drop in different styles. The curves with relatively smaller defect sizes (i.e., $d=0.125 l, 0.25 l)$ drop sharply while the curves with relatively larger defect sizes (i.e., $d=0.5 l, 0.75 l$ ) drop moderately. It suggests that the damage mode changes from the brittle fracture to ductile fracture as the defect size is increased.

In order to check the validity and feasibility of the present numerical method for introducing defects, the computational predictions of the normalized peak loads are compared with the existing experimental results [16], as shown in Fig. 4, the value $F_{p}^{0}$ denotes the peak load for the case without defect. Overall, the present computational results agree well with those measured, thus the present numerical method is suitable for the study of defects.

\subsection{Influence of defect location}

In this section, the influence of the defect location is investigated and a single defect is accordingly considered for simplification. As shown in Fig. 1b, a length variable $b$, representing the distance between the left extremity of the adhesive layer and the symmetry axis of the defect, is introduced to describe the location of the defect.

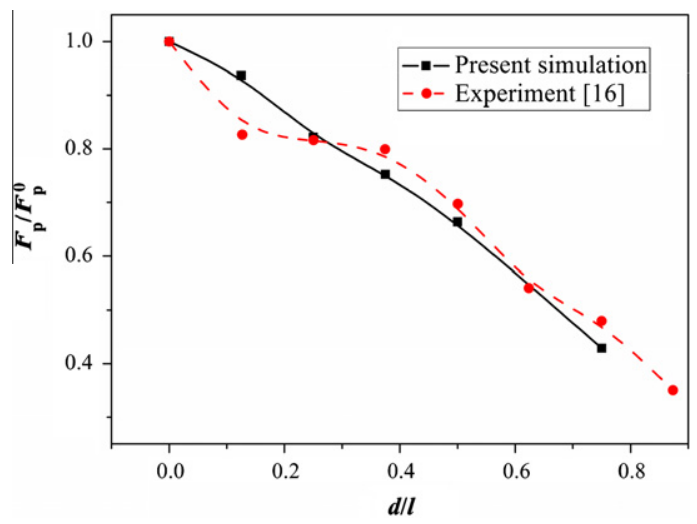

Fig. 4. Normalized peak load plotted as the function of normalized defect sizes $d / l$ : comparison between the present model predictions with the experimental measurements [16].
Fig. 5 plots the normalized load as a function of displacement for the cases considering the defect location for selected defect sizes $d$ : (a) $0.125 l$; (b) $0.25 l$; (c) $0.375 l$; (d) $0.5 l$ and (e) $0.75 l$. All the curves in Fig. 5 consist of the rising part and declining part. Differently, when the defect size is relatively smaller (e.g., $d=0.125 l$ shown in Fig. 5a), the load-displacement curves decline sharply. On the contrary, when the defect size is relatively larger (e.g., $d=0.75 \mathrm{l}$ shown in Fig. 5e), the curves decline slowly. Based on the results of Fig. 5, the peak load is plotted as a function of the length $b$ for selected defect sizes, as shown in Fig. 6, so as to present the influence of the defect location on the load-bearing capability. Generally, with the varying of the defect location, the variation of normalized peak load is slight. In other words, the influence of defect location on the peak load is negligible.

However, it should be mentioned that the influence of the special defect size should be considered when the defect location is studied. When the joint is stretched, the normal and shear stress in the adhesive attain to high values at the overlap extremities and very low values in the central region of the overlap. The central region with the low stress values has a length of some value. Accordingly, when the defect size is small (e.g., $d=0.125 l$ ), the defect on the mid-region would influence the stress distribution in the adhesive less significantly compared to that close to one overlap extremity. Therefore, the peak load declines slightly as the defect is located close to the overlap extremity, with the defect size of $0.125 l$ (see, Fig. 6). By contrast, when the defect size is large (e.g., $d=0.75 l$ ), even larger than the length of central region with the low stress values, the defect on the mid-region would influence the stress distribution in the adhesive more remarkably than the case of small defect. Consequently, it is not obvious to judge that which case will induce low joint strength by comparison between the cases with the defect on mid-region and on overlap extremity. Therefore it is acceptable that the peak load increases slightly as the defect is located close to one overlap extremity, with the defect size of $0.75 l$ (see, Fig. 6).

\section{Weak bonding}

In contrast to local debonding, weakly bonded joints show no sign of separation in the bondline [11]. In other words, a weakly bonded joint is still bonding the two adherends together, which is shown in Fig. 1b. In practice, weak bonding may be caused by many reasons, such as the use of heterogeneous glue and rough bonding surface. In this section, the influence of weak bonding is investigated by the numerical model described below.

\subsection{Model description}

As presented in Fig. 1b, the overlap of the model considering weak bonding is similar to that considering local debonding. Differently, the local debonding is replaced by the weak bonding with assigning the prescribed material parameters. Generally, the local debonding is a special kind of weak bonding with the material parameters having zero-value. Accordingly, the present model is similar to the model considering local debonding except the material parameters of defect. It has been established that whilst the peak value and area of the T-S curve are vital for capturing the interface separation behavior [31]. In other words, the separation strength and fracture energy are two sensitive parameters to describe the fracture properties of adhesives. Thus, the weak bonding can be regarded as the case that the values of the interface parameters are lower in some region of the adhesive layer. The present simulation only considers the case with the weak bonding locating at the mid-region of the adhesive layer. The weak bonding are hence determined by the equations below, 

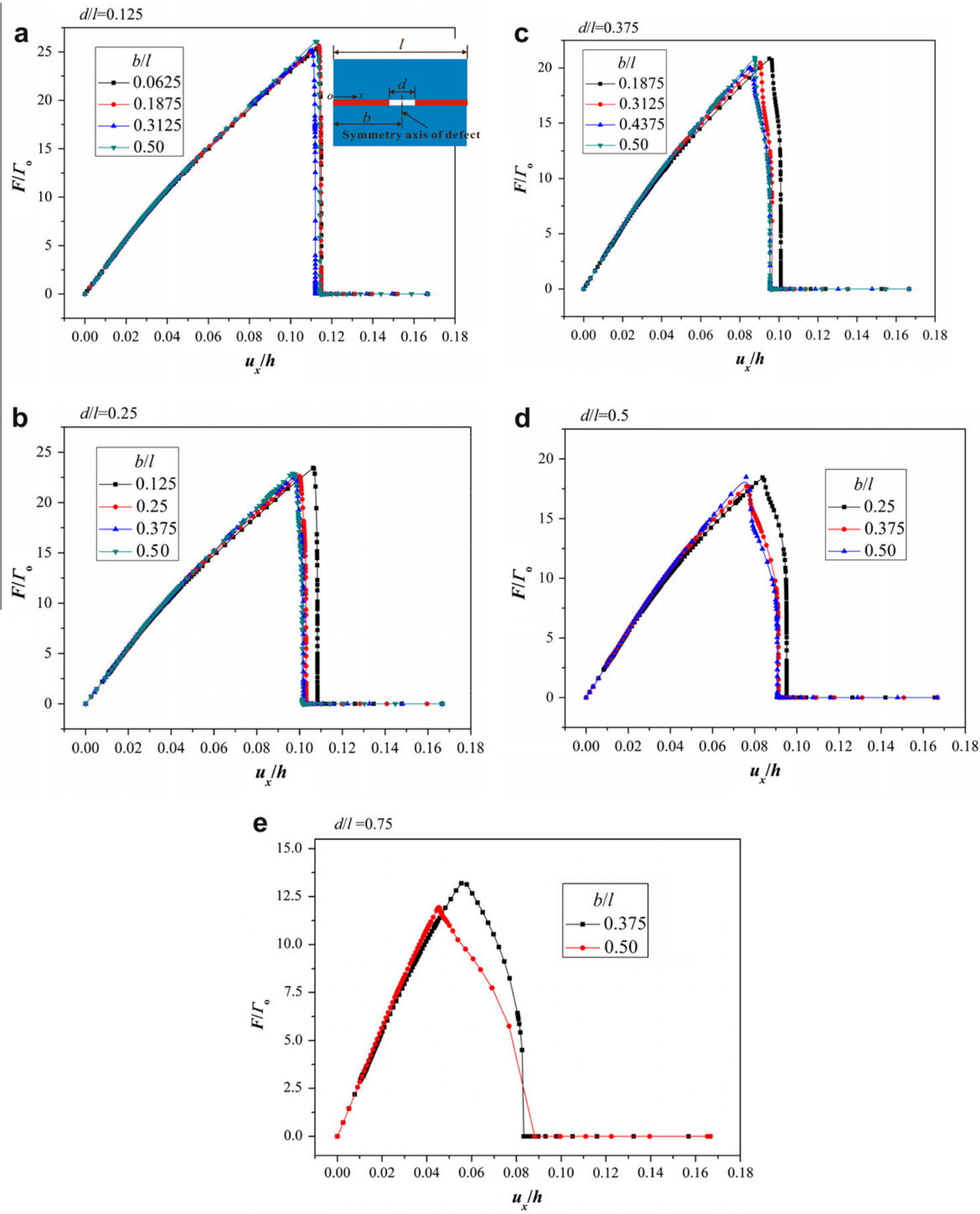

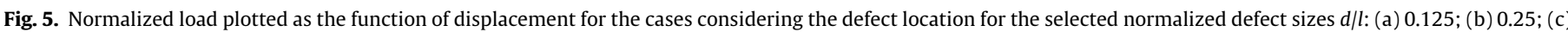
0.375 ; (d) 0.5 ; and (e) 0.75 .

$\Gamma^{d}=\Gamma^{d}(x)= \begin{cases}p \Gamma_{0} & x \in\left(b-\frac{1}{2} d, b+\frac{1}{2} d\right) \\ \Gamma_{0} & x \notin\left(b-\frac{1}{2} d, b+\frac{1}{2} d\right)\end{cases}$

$\sigma_{a d}^{d}=\sigma^{d}(x)= \begin{cases}q \sigma_{a d} & x \in\left(b-\frac{1}{2} d, b+\frac{1}{2} d\right) \\ \sigma_{a d} & x \notin\left(b-\frac{1}{2} d, b+\frac{1}{2} d\right)\end{cases}$

where $\Gamma^{d}$ and $\sigma_{a d}^{d}$ represent the fracture energy and separation strength of the weak bonding. In order to describe the weaken degree of the defect, weakening coefficients $p$ and $q$ are introduced, varying between 0 and 1 . Obviously, the weaken degree becomes higher as the weakening coefficients are lower. Then defect degrades to the local debonding with the two weakening coefficients of 0 , while the defect disappears with the coefficients of 1 .

\subsection{Results}

As mentioned in Section 3.2, peak load could be used to assess the load-bearing capability of the adhesively bonded joints. Fig. 7 shows the normalized peak load plotted as a function of normalized separation strength of the adhesive in weak bonding area, considering various adhesive fracture energies $(\Gamma)$ and sizes $(d)$ of the weak bonding region. Fig. 7 presents the variation of load- 


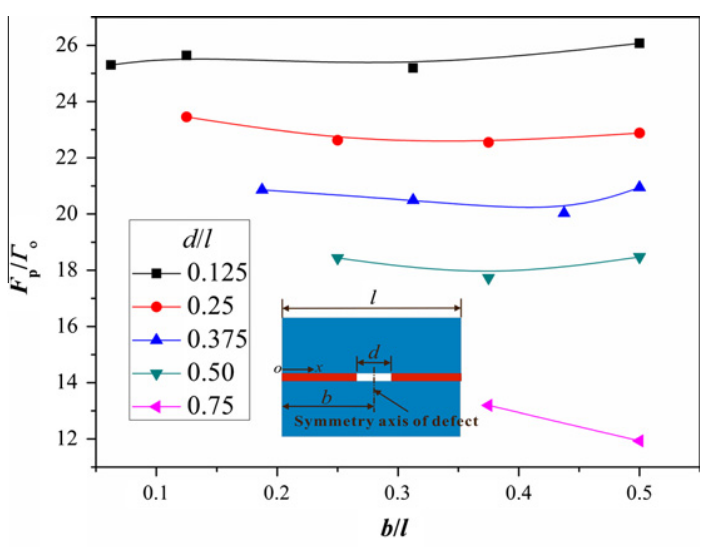

Fig. 6. Normalized peak load plotted as a function as location variable for the selected defect sizes.

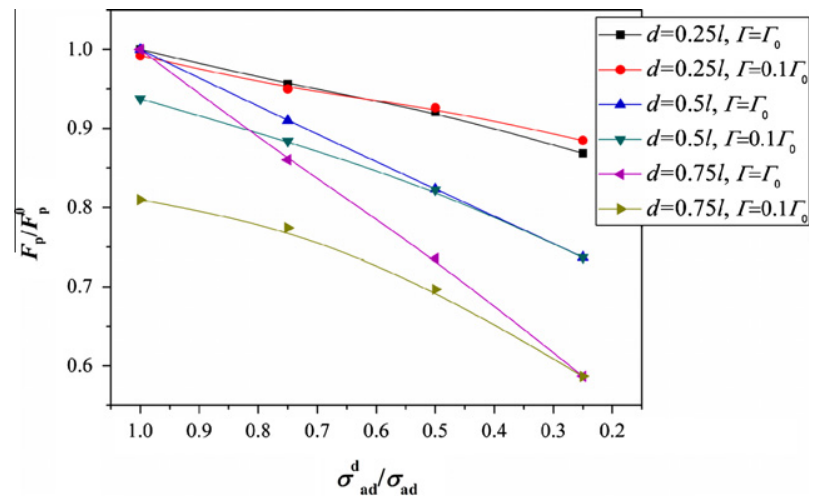

Fig. 7. Normalized peak load plotted as a function as normalized separation strength of the adhesive in weak bonding region, considering various adhesive fracture energies and sizes of the weak bonding region.

bearing capability due to the defect. All the curves in this figure decline as the separation strength of the weak adhesive is decreased. It should be mentioned the influence of the fracture energy, two selected values of which are considered, namely $\Gamma=\Gamma_{0}$ and $\Gamma=0.1 \Gamma_{0}$. When the size of the defect is small (i.e., $d=0.25 l$ ), difference between the curves corresponding to the case of $\Gamma=\Gamma_{0}$ and the case of $\Gamma=0.1 \Gamma_{0}$ is negligible. However, when the size of the defect is large (i.e., $d=0.75 l$ ), the difference of that is noticeable. The peak load corresponding to the case of $\Gamma=\Gamma_{0}$ is higher than that corresponding to the case of $\Gamma=0.1 \Gamma_{0}$, which is more remarkable when $\sigma_{a d}^{d}$ is relatively larger. In addition, when $\sigma_{a d}^{d}$ is small enough, namely $\sigma_{a d}^{d} / \sigma_{a d}=0.25$, the values of the peak load corresponding to the two cases are extremely close.

\section{Void}

Besides strip defects, void-like defect is another common type of defect in adhesive, which arises both from volatile by-products that can evolve during the adhesive curing or from entrapped air bubbles [36]. In this section, an alternative approach employing modified Gurson model is carried out to simulate the adhesive with voids.

\subsection{Interface elements}

Gurson model was established for porous materials which depend on the void volume fraction as an additional internal variable [32]. The model was further developed by Tvergaad with respect to the yield condition and damage evolution [33,34] and by Needleman to include the law of void nucleation [35]. Therefore, the Gurson model used in present simulation can also be referenced as Gurson-Tvergaad-Needleman (GTN) model, which is an isotropic formulation that employs the invariants of the Cartesian components of the Cauchy stress (i.e., $\sigma_{i j}$ ). The yield function of the GTN model is specified by:

$\Phi=\left(\frac{\sigma_{\mathrm{eq}}}{\sigma_{0}}\right)^{2}+2 q_{1} f \cosh \left(\frac{3 q_{2} \sigma_{\mathrm{m}}}{2 \sigma_{0}}\right)-\left[1+\left(q_{1} f\right)^{2}\right]=0$

where $\sigma_{\text {eq }}$ denotes the von Mises stress, $\sigma_{\text {eq }}=\sqrt{3 s_{i j} s_{i j} / 2}$, with $s_{i j}$ representing stress deviator (i.e., $s_{i j}=\sigma_{i j}-1 / 3 \sigma_{k k} \delta_{i j}$ ). And $\sigma_{m}$ denotes the hydrostatic stress (i.e., $\sigma_{m}=\sigma_{k k} / 3$ ); $\sigma_{0}$ represents the yield strength of the adhesive without defect in the present simulation; $q_{1}$ and $q_{2}$ are the fitting parameters with the given values, namely, $q_{1}=1.5$ and $q_{2}=1.0[33,36]$. The current state is characterized by the damage parameter $f$, which can be interpreted as the void volume fraction.

In the GNT model, as the deformation of the adhesive is increased, the void volume fraction would increase, which is caused by two factors, namely, void nucleation and void growth. They are both controlled by mean plastic strain $\varepsilon_{k k}^{p}$. The current value of the void volume fraction changes due to the growth of existing voids and the nucleation of new void, which can be expressed by:

$\dot{f}=\dot{f}_{\text {growth }}+\dot{f}_{\text {nucleation }}=(1-f) \varepsilon_{k k}^{p}+A \bar{\varepsilon}^{P}$

where $\bar{\varepsilon}^{P}$ is the equivalent plastic strain and $\varepsilon_{k k}^{p}$ is the hydrostatic component of the plastic strain. $A$ is specified by,

$A=\frac{f_{N}}{S \sqrt{2 \pi}} \exp \left[-\frac{1}{2}\left(\frac{\bar{\varepsilon}^{P}-\varepsilon_{N}}{S}\right)^{2}\right]$

where $f_{N}$ is the volume fraction of the nucleated void, the nucleation function $A / f_{N}$ agrees with the normal distribution with a mean value $\varepsilon_{N}$ and standard deviation $S$. Both of them have the value of 0.1 in the investigation.

In this simulation for voids, the interface elements are regarded as a single layer of four-node plane strain elements with the yield function fitting the GTN model described above. The interface elements have thickness and a typical value of adhesive thickness (i.e., $0.5 \mathrm{~mm}$ ) is selected in the simulation. The interface elements lose their connecting capability totally (i.e., total failure) when the value of $f$ reaches 1 .

\subsection{Influence of void size}

Various initial void fractions are explored, for the purpose of studying the influence of voids on the load-bearing capacity, with the result shown in Fig. 8, all the curves in this figure present the similar trend, the load rises up to a peak value and declines subsequently as the displacement is increased. With the increasing of the initial void fraction, the load-displacement curves exhibit significant discrepancy. With the initial void fraction increasing from 0 (i.e., the case without defect) to 0.6 , the peak load reduces by nearly $2 / 3$ times. For the latter case, the joint lost its load-bearing capacity totally when the normalized displacement increases to 0.073 , which could be regarded as the failure displacement. It is reasonable to predict that the failure displacement would be larger according to the tendency of the plots for other cases. Fig. 8 implies the strength of the joints has strong size-scale dependence of the initial void fraction in the adhesive layer. 


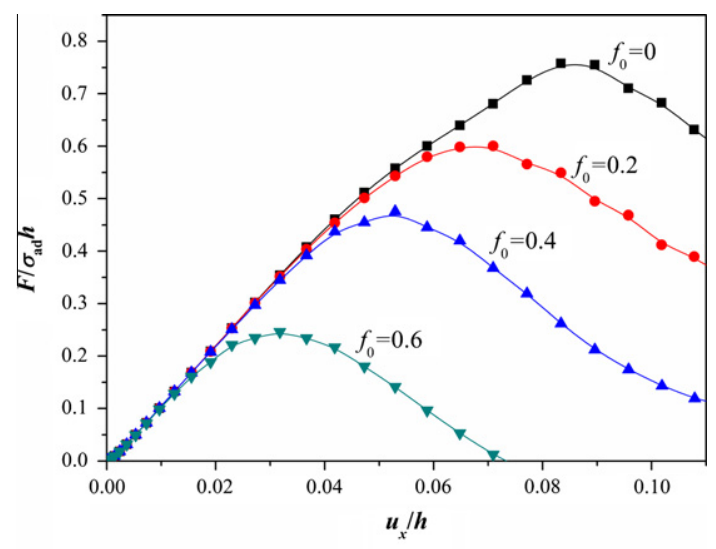

Fig. 8. Load-displacement relations for selected initial void fractions.

\section{Concluding remarks}

In summary, the method of finite element has been utilized to study the strength of metallic lap joints with defects in their adhesive layers. Three types of defects are taken into account respectively, which are local debonding, weak bonding and void. For the first two types of defects, a developed numerical method using the cohesive zone model modified by user-defined subroutines is carried out as to consider the influences of the defect size and location. Furthermore, the GTN model is employed to simulate the adhesive with voids, considering the influence of the void size. The results show that the overall strength of the joints diminishes as the defects size is increased. Especially, the adhesive fracture properties and the size of weak bonding region have combined influences on the strength of the joints. The residual strength of joints is significantly influenced by fracture properties of the weak adhesive when the weakly bonded area is relatively larger. Obtained results provide fundamental principles for factual strength assessment of adhesively bonded joints with defects in their adhesives.

It should be noted that in the present research, several assumptions and simplifications are adopted, which would cause the deviation between the simulation and practice. Firstly, three types of defects are considered separately. Combined influence of multitype defects should be investigated in the future. Secondly, both the shape and the distribution of the defects in the present model are regular, while the situations of the defects in practice are random. More sophisticated models are certainly needed to study the effects of random defects. Thirdly, Generally speaking, the adhesive thickness could influence the strength of the adhesively bonded joints, previous researches have studied the influence and shown some results [37]. The present investigation aim to study the influence of defects, the influence of adhesive thickness is thence not considered. CZM could be regarded as an equivalent approach to simulate the adhesive. It has been established that the separation strength (i.e., peak traction) and fracture energy are vital for capturing the interface separation behavior when the CZM are employed. Since the previous researches have proposed that the two important cohesive parameters varied as functions of adhesive thickness [38], the variation of adhesive thickness could be implemented by introducing different cohesive parameters in the future simulation.

\section{Acknowledgment}

This work was supported by the National Natural Science Foundation of China (11021262, 10932011 and 90816004).

\section{References}

[1] R. Kahraman, M. Sunar, B. Yilbas, Journal of Materials Processing Technology 205 (2008) 183.

[2] Z.S. Wu, H. Yuan, H.D. Niu, Journal of Engineering Mechanics-ASCE 128 (2002) 562.

[3] J.G. Dai, T. Ueda, Y. Sato, Journal of Composites for Construction 9 (2005) 52.

[4] F. Ascione, Composites Part B-Engineering 40 (2009) 116.

[5] L.D.R. Grant, R.D. Adams, L.F.M. da Silva, International Journal of Adhesion and Adhesives 29 (2009) 405.

[6] L.D.R. Grant, R.D. Adams, L.F.M. da Silva, International Journal of Adhesion and Adhesives 29 (2009) 535.

[7] A.L. Loureiro, L.F.M. da Silva, C. Sato, M.A.V. Figueiredo, Journal of Adhesion 86 (2010) 765.

[8] A. Higgins, International Journal of Adhesion and Adhesives 20 (2000) 367.

[9] M.F.S.F. de Moura, R. Daniaud, A.G. Magalhaes, International Journal of Adhesion and Adhesives 26 (2006) 464.

[10] J.H. Park, J.H. Choi, J.H. Kweon, Composite Structures 92 (2010) 2226.

[11] R.B. Heslehurst, International Journal of Adhesion and Adhesives 19 (1999) 133.

[12] S. Yang, L. Gu, R.F. Gibson, Composite Structures 51 (2001) 63.

[13] L. Llopart, K.I. Tserpes, G.N. Labeas, Composite Structures 92 (2010) 1673

[14] K.I. Tserpes, S. Pantelakis, V. Kappatos, Computational Materials Science 50 (2011) 1372.

[15] N.G. Berry, J.R.M. d'Almeida, Polymer Testing 21 (2002) 373.

[16] J.D. Engerer, E. Sancaktar, International Journal of Adhesion and Adhesives 31 (2011) 373.

[17] M. Olia, J.N. Rossettos, International Journal of Solids and Structures 33 (1996) 2681.

[18] A. Chadegani, R.C. Batra, International Journal of Adhesion and Adhesives 31 (2011) 455.

[19] C.L. Chow, C.W. Woo, Theoretical and Applied Fracture Mechanics 4 (1985) 75.

[20] M. You, Z.M. Yan, X.L. Zheng, H.Z. Yu, Z. Li, International Journal of Adhesion and Adhesives 27 (2007) 696.

[21] F.L. Ribeiro, L. Borges, J.R.M. d'Almeida, International Journal of Adhesion and Adhesives 31 (2011) 331.

[22] T. Pardoen, T. Ferracin, C.M. Landis, F. Delannay, Journal of the Mechanics and Physics of Solids 53 (2005) 1951.

[23] A. Rudawska, International Journal of Adhesion and Adhesives 30 (2010) 574.

[24] N. Chandra, H. Li, C. Shet, H. Ghonem, International Journal of Solids and Structures 39 (2002) 2827.

[25] L.B. Freund, S. Suresh, Thin Film Materials: Stress, Defect Formation and Surface Evolution, Cambridge University Press, Cambridge, 2003.

[26] S. Ghosh, Y. Ling, B. Majumdar, R. Kim, Mechanics of Materials 32 (2000) 561.

[27] H. Li, N. Chandra, International Journal of Plasticity 19 (2003) 849.

[28] C. Balzani, W. Wagner, Engineering Fracture Mechanics 75 (2008) 2597.

[29] M.F.S.F. de Moura, R.D.S.G. Campilho, J.P.M. Goncalves, Journal of Adhesion Science and Technology 23 (2009) 1477.

[30] A. Derewonko, J. Godzimirski, K. Kosiuczenko, T. Niezgoda, A. Kiczko, Computational Materials Science 43 (2008) 157.

[31] A.G. Evans, J.W. Hutchinson, Y. Wei, Acta Materialia 47 (1999) 4093.

[32] A.L. Gurson, Journal of Engineering Materials and Technology - Transactions of the ASME 99 (1977) 2.

[33] V. Tvergaard, International Journal of Fracture 18 (1982) 237.

[34] V. Tvergaard, A. Needleman, Acta Metallurgica 32 (1984) 157.

[35] A. Needleman, V. Tvergaard, International Journal of Fracture 49 (1991) 41

[36] V. Tvergaard, International Journal of Fracture 17 (1981) 389.

[37] L.F.M. da Silva, T.N.S.S. Rodrigues, M.A.V. Figueiredo, M.F.S.F. de Moura, J.A.G. Chousal, Journal of Adhesion 82 (2006) 1091.

[38] T. Carlberger, U. Stigh, Journal of Adhesion 86 (2010) 814. 